\title{
Sex-differential effects of olanzapine vs. aripiprazole on glucose and lipid metabolism in first-episode schizophrenia
}

\author{
Xue-Mei ZHou1 \\ http://orcid.org/0000-0002-4774-4432 \\ MaO-Rong Hu2 \\ http://orcid.org/0000-0002-8541-2893 \\ MeI-Yu Gong ${ }^{1}$ \\ http://orcid.org/0000-0002-9092-3006 \\ Xue-Liang Zou ${ }^{1}$ \\ http://orcid.org/0000-0003-0688-1550 \\ ZHI-MiN Yu ${ }^{1}$ \\ http://orcid.org/0000-0002-8732-4811 \\ 1 Jiangxi Mental Health Centre, Nanchang, Jiangxi, China. \\ 2 The First Affiliated Hospital of Nanchang University, Nanchang, Jiangxi, China.
}

Received: 06/29/2018 - Accepted: 04/02/2019

DOl: 10.1590/0101-60830000000189

\begin{abstract}
Objective: To compare sex difference in metabolic effect of olanzapine versus aripiprazole on schizophrenia. Methods: A twelve-week prospective open-label cohort study to compare four subgroups according to first-episode schizophrenia patients' type of drug usage and sex: female aripiprazole $(\mathrm{n}=11)$, male aripiprazole $(\mathrm{n}=11)$, female olanzapine $(\mathrm{n}=10)$, and male olanzapine $(\mathrm{n}=11)$ for body mass index, fasting serum triglyceride, total cholesterol, high-density lipoprotein cholesterol, low-density lipoprotein cholesterol, and fasting glucose. Results: Aripiprazole may be associated with weight gain in female patients with low-baseline weight. Aripiprazole may have an adverse effect of weight and favorable effects of circulating glucose and lipid on female over male schizophrenia patients. The aripiprazole-induced changes in glucose and lipid may be independent of body fat storage, especially for female schizophrenia patients. Olanzapine may have adverse effects of weight, glucose and lipid profiles on female over male schizophrenic patients. Discussion: Our findings fill the gap in knowledge and provide a sex-specific guidance to psychiatrist better tailoring treatment to individual sex-differential characteristics and a key clue to understand the sex-differential mechanism of antipsychotics-induced metabolic dysfunction.
\end{abstract}

ZHou XM et al. / Arch Clin Psychiatry. 2019;46(2):33-9

Keywords: Schizophrenia, sex difference, glucose and lipid, olanzapine, aripiprazole.

\section{Introduction}

The US National Institutes of Health (NIH) has been urging identifying sex-specific risks of adverse events in all NIH-funded schizophrenia-related researches ${ }^{1}$. Some individual prevalences of metabolic syndrome (MS) components differed statistically between male and female schizophrenia patients. Lots of researches in schizophrenia patients treated with antipsychotics had reported females with higher prevalence of abnormally low high-density lipoprotein cholesterol (HDL) than that of males ${ }^{2-7}$, and the latter with higher prevalence of high triglyceride level over the former $2,6-8$. Rezaei et al. had claimed not only low HDL but also high triglyceride level of female chronic schizophrenia patients was more prevalent than that of male counterparts ${ }^{9}$. A recent study from China had found higher low-density lipoprotein cholesterol (LDL) (but not HDL and triglyceride) plasma levels of female than male chronic schizophrenia patients ${ }^{10}$. Another recent study without reporting specific antipsychotic drug and time span of antipsychotic treatment had found higher levels of serum triglyceride and LDL as well as lower level of HDL in male first-episode schizophrenia patients from China than female counterparts ${ }^{11}$. Most researchers did not find sex difference in prevalence of fasting glucose, except for two studies, ${ }^{6,9}$ reporting higher prevalence of female schizophrenia patients with elevated glucose level meeting specific blood glucose criterion of MS than that of male counterparts. Despites highly prevalent metabolic adverse effects among schizophrenic patients taking antipsychotics, the sex-specific data on antipsychotics-induced dyslipidemia and impaired glucose metabolism in schizophrenia patients are preliminary at best, and show conflicting results. It is therefore imperative to indentify sex-differential effects of second-generation antipsychotic drugs (SGAs) on glucose and lipid metabolism, which is instructive to add some sex-specific therapeutic strategies into future clinical psychiatry practice. Among commonly used SGAs, undoubtedly, olanzapine is one of the most potential drugs inducing weight gain, glucose metabolism abnormalities and/or hyperlipidemia, whereas aripiprazole with minimal or no effect on glucose and lipid metabolism and the lowest weight-gain liability. Therefore, we focus on a sex-differential effect of either olanzapine or aripiprazole on body weight and glucose and lipid profiles in a population of antipsychotic-naïve first-episode schizophrenia patients with monotherapy after hospitalization, and raise the following questions: During the first trimester of antipsychotic treatment, (1) what were the respective changes in metabolic parameters between the sexes and between two drugs from baseline to endpoint? (2) what was the interaction between weight gain and the changes in glucose and lipid level?

\section{Methods and materials}

The study was approved by Jiangxi Mental Health Centre Ethics Committee, China, in accordance with the Declaration of Helsinki ${ }^{12}$. A written informed consent was obtained from all study participants after the nature of the procedures had been fully explained. 


\section{Participants}

Participant enrollment began in February 2015 and ended in October 2015. A total of forty-three participants (twenty-two men and twentyone women) aged 18-48 years were the inpatients from the psychiatric units in Jiangxi Mental Health Centre, China. These people were experiencing their first psychotic episode of schizophrenia diagnosed in accordance with criteria established in DSM-V ${ }^{13}$ and had not previously received any antipsychotic drug treatment.

The exclusion criteria included: any psychiatric disorder other than schizophrenia (according to DSM-V); history of any substance-related and addictive disorders met DSM-V criteria; a significant risk of committing suicide based on history or mental status exam; pregnant or lactating patients; specific systemic diseases; or other medical conditions such as diabetes mellitus, dyslipidemia, cardiovascular diseases, and hypertension; clinically significant abnormal laboratory test results, vital sign, or electrocardiogram findings.

\section{Study design}

Aripiprazole (Zhejiang Otsuka Pharmaceutical Co. Ltd., China, mean $20.0 \mathrm{mg}$ /day) or olanzapine (Lilly del Caribe Inc., USA, mean 15.4 $\mathrm{mg} /$ day) monotherapy was not randomized but provided according to normal local clinical practice. Participants were divided into four groups according to their type of drug usage and sex: female aripiprazole (FA), male aripiprazole (MA), female olanzapine (FO), and male olanzapine (MO). The primary concerns in present study were the comparison on mean changes in metabolic parameters from the baseline (i.e., prior to starting either aripiprazole or olanzapine treatment) to the fourth, eighth, and twelfth weekend after starting the treatment in the four cohorts, including the body mass index (BMI), triglyceride (TG), total cholesterol (TC), high density lipoprotein cholesterol (HDL), low density lipoprotein cholesterol (LDL), and fasting glucose (FG).

All forty-three participants remained hospitalized for the entirety of their participation to observe uniform clinical and environmental characteristics of the cohorts. They received dietetically balanced hospital meals (daily energy intake: men $10.5 \mathrm{MJ}$ (2,500 kcal), women $9.2 \mathrm{MJ}(2,200 \mathrm{kcal}))$, had occasionally access to meals brought by relatives other than being required to attend group, and had the opportunity for two hours' physical exercise each day. No patient was concurrently treated with any other drug known to affect serum glucose or lipid concentrations or appetite throughout the whole study period, also at least one continuous 6-month period before enrollment. Only trihexyphenidyl for extrapyramidal symptoms or lorazepam for insomnia or agitation was allowed as needed.

\section{Assessments}

Height and weight were measured, and BMI was calculated for all patients. All patients were rated using the Positive and Negative Syndrome Scale ${ }^{14}$ for their clinical psychotic symptoms by the senior psychiatrist trained in its use, with an acceptable minimum score of sixty on admission.

\section{Laboratory analyses}

Whole venous blood samples were drawn at the early morning of the study days after overnight fasting. The fasting serum glucose and lipids were analyzed by enzymatic procedure for TC, TG and FG, by direct measurement for both HDL and LDL applying the AU680 Chemistry Analyzer (Beckman Coulter, Inc. USA) in the standard clinical biochemistry laboratory.

\section{Statistical analyses}

Descriptive statistics were computed for the basic demographic and clinical variables. A post hoc longitudinal repeated-measures analyses were performed for the mean change from baseline to each specified postbaseline visit in the dependent variable (i.e., BMI, glucose and lipid) measures between-drug and within-drug, as well as between-sex and within-sex with Generalized Additive Mixed Model (GAMM), an ideal method analyzing non-normal longitudinal data given the fact that the residuals, but not the dependent variable, are required to meet the Gaussian distribution in this model. In GAMM, the main effect of treatment time, the interaction terms of either treatment time $\times$ antipsychotic drug or treatment time $\times$ sex on the evolution of continuous metabolic parameters were evaluated. Because the residual analysis of the raw datum for BMI failed to met the Gaussian distribution, we took the normalized data into the GAMM. The statistical powers of the different sample size drawing different inference were presented in Stable 1 to Stable 2 from online supplementary content (available upon request to the authors).

All analyses were performed using Empower (R) (www.empowerstats.com, X and Y solutions, inc. Boston MA) and R (http://www.R-project.org) at $\mathrm{P} \leq 0.05$ levels (two-sided).

Given the crucial effect of the sample size on a test of significance in any medical study, we have believed that any inference could come not only from data with statistically significant difference, but also from data with regular, resultantly discernible and traceable pattern of change in effect size, despite no statistical significance.

\section{Results}

The progress of participants through the study was shown in Figure 1. Treatment groups were similar with respect to baseline demographic and clinical variables (Table 1).

\section{Body mass index (BMI)}

The FA group showed the magnitude significantly increasing in the NBMI at every postbaseline visit than baseline in patients with aripiprazole. The significant between-sex difference in baseline NBMI meant more body weight of MA than FA before aripiprazole treatment (Table 2). However, the increase in NBMI of FA overtook that of MA month by month during the postbaseline visits (Figure 2), meaning aripiprazole-induced weight gain of FA more than that of MA. Taken together, these data demonstrate that aripiprazole may not only be associated with weight gain in female schizophrenia patients with low baseline weight, but also have a more adverse effect of weight on female patients than male patients.

Table 1. Baseline demographic and clinical characteristics among 43 first-episode schizophrenia patients assigned to 12 weeks treatment with aripiprazole or olanzapine

\begin{tabular}{|l|c|c|c|}
\hline & $\begin{array}{c}\text { Aripiprazole } \\
(\mathrm{n}=22)\end{array}$ & $\begin{array}{c}\text { Olanzapine } \\
(\mathrm{n}=21)\end{array}$ & $p$ value \\
\hline Age (years) & $24.18 \pm 7.04$ & $28.24 \pm 10.57$ & 0.144 \\
\hline Sex, $\mathrm{n}(\%)$ & \multicolumn{3}{|l|}{} \\
\hline Male & $11(50.0 \%)$ & $11(52.4 \%)$ & \\
\hline Female & $11(50.0 \%)$ & $10(47.6 \%)$ & \\
\hline Duration of disease (months) & $20.52 \pm 19.66$ & $14.63 \pm 21.01$ & 0.35 \\
\hline PANSS scores & $99.95 \pm 10.04$ & $104.29 \pm 25.83$ & 0.47 \\
\hline BMl & $22.0 \pm 4.75$ & $21.11 \pm 2.77$ & 0.46 \\
\hline Triglyceride concentration (mmol/L) & $0.80 \pm 0.34$ & $0.82 \pm 0.32$ & 0.88 \\
\hline Total cholesterol concentration (mmol/L) & $4.24 \pm 0.79$ & $4.02 \pm 0.79$ & 0.35 \\
\hline LDL concentration (mmol/L) & $2.22 \pm 0.73$ & $1.97 \pm 0.51$ & 0.22 \\
\hline HDL concentration (mmol/L) & $1.53 \pm 0.37$ & $1.51 \pm 0.42$ & 0.85 \\
\hline FG concentration (mmol/L) & $5.05 \pm 0.64$ & $5.13 \pm 0.57$ & 0.65 \\
\hline
\end{tabular}

Data are mean (SD) or $n(\%)$. PANSS: the Positive and Negative Syndrome Scale. BMI: body mass index; LDL: Iow-density lipoprotein cholesterol; HDL: high-density lipoprotein cholesterol; FG: fasting serum glucose. 


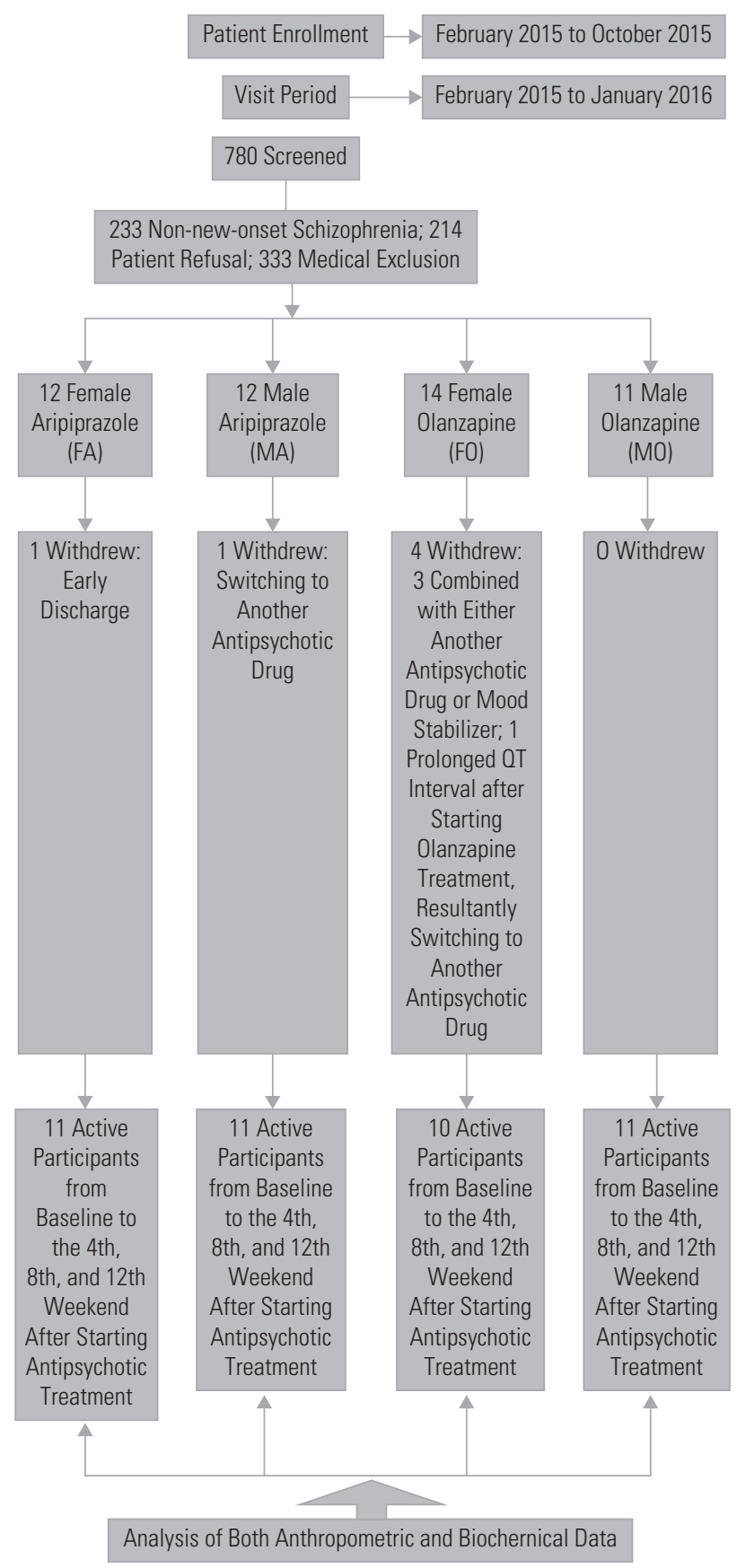

Figure 1. Participant Recruitment and Visit Flow Diagram.
The significant between-sex difference in baseline NBMI meant more body weight of $\mathrm{MO}$ than that of FO before olanzapine treatment. During the postbaseline visits, however, the weight gain of FO significantly exceeded that of MO in turn (Table 3, Figure 2). It is thereby demonstrated that olanzapine may have a more adverse effect of weight on female patients than male patients.

\section{Serum lipids}

\section{Triglyceride (TG)}

In view of the smallest magnitude increasing in FA's NTG compared to the other three subgroups, despite its difference respectively reaching statistic significance at fourth and twelfth weekend than baseline, the effect of aripiprazole on NTG was still considered favourable in female patients with schizophrenia. The gradually increased between-sex differences in NTG from baseline to postbaseline visits in patients with aripiprazole showed more NTG gain of the males than that of the females over treatment time (Table 2, Figure 2). Taken together, these data demonstrate that aripiprazole may have a favourable effect of TG on female over male patients with schizophrenia.

The FO group showed the magnitude significantly increasing in the NTG at every postbaseline visit than baseline in patients with olanzapine. With the progress of treatment, the between-sex difference in NTG was made by males more than females at baseline into females over males during postbaseline visits. Taken together, these data reflect a more adverse effect of olanzapine on TG in female patients than that of male patients (Table 3, Figure 2).

The increasing difference in MA's NTG at every postbaseline visit than baseline was much more numerical than the counterpart of MA's NBMI in the male patients (Table 4).

\section{Low density lipoprotein cholesterol (LDL)}

The differences decreasing mildly in FA's NLDL at any postbaseline visit than baseline resulted in positive between-sex differences in NLDL throughout the whole study period in patients with aripiprazole (Table 2, Figure 2), meaning aripiprazole may have a favourable effect of LDL on female over male patients with schizophrenia.

The FO group showed the significant increases in postbaseline NLDL than baseline so that the magnitude increasing in MO's NLDL were less than that of FO group in patients with olanzapine (Table 3, Figure 2), reflecting olanzapine may have an adverse effect of LDL on female over male patients with schizophrenia.

\section{Total cholesterol (TC)}

The numerically decreasing differences in postbaseline FA's NTC than baseline caused gradually increased between-sex differences in NTC at any postbaseline visit than baseline in patients with aripiprazole (Table 2, Figure 2), demonstrating aripiprazole may have a favourable effect of TC on female over male patients with schizophrenia.

Table 2. The between-sex and within-sex evolution of the individual metabolic parameter over time in patients with first-episode schizophrenia assigned to 12 weeks treatment with aripiprazole

\begin{tabular}{|l|c|c|c|c|c|c|}
\hline & NBMI & NTG & NLDL & NTC & NHDL & NFG \\
\hline Baseline, female & $-1.07(-1.61--0.53)$ & $-0.81(-1.30--0.31)$ & $-0.23(-0.81-0.34)$ & $-0.03(-0.59-0.53)$ & $0.52(-0.02-1.05)$ & $0.20(-0.42-0.83)$ \\
\hline (The 4th weekend - Baseline), female & $0.35(0.18-0.52)$ & $0.66(0.21-1.11)$ & $-0.21(-0.65-0.22)$ & $-0.22(-0.63-0.19)$ & $-0.32(-0.76-0.13)$ & $-0.52(-1.16-0.12)$ \\
\hline (The 8th weekend - Baseline), female & $0.53(0.36-0.70)$ & $0.39(-0.06-0.84)$ & $-0.08(-0.52-0.35)$ & $-0.18(-0.59-0.24)$ & $-0.23(-0.67-0.21)$ & $-0.58(-1.23-0.06)$ \\
\hline (The 12th weekend - Baseline), female & $0.75(0.58-0.92)$ & $0.59(0.14-1.04)$ & $-0.06(-0.50-0.37)$ & $-0.27(-0.69-0.14)$ & $-0.13(-0.57-0.31)$ & $-0.41(-1.05-0.23)$ \\
\hline Baseline $\times$ (male - female) & $1.49(0.72-2.25)$ & $0.14(-0.56-0.84)$ & $0.19(-0.62-1.00)$ & $-0.34(-1.12-0.45)$ & $-0.75(-1.50-0)$ & $-0.07(-0.95-0.82)$ \\
\hline The 4th weekend $\times$ (male - female) & $-0.19(-0.43-0.05)$ & $0.04(-0.60-0.68)$ & $0.16(-0.45-0.78)$ & $0.31(-0.27-0.89)$ & $0.37(-0.26-0.99)$ & $0.01(-0.90-0.92)$ \\
\hline The 8th weekend $\times$ (male - female) & $-0.27(-0.51-0.03)$ & $0.56(-0.07-1.20)$ & $0.06(-0.55-0.68)$ & $0.38(-0.20-0.96)$ & $0.27(-0.35-0.90)$ & $0.03(-0.88-0.94)$ \\
\hline The 12th weekend $\times$ (male - female) & $-0.28(-0.52--0.04)$, & $0.62(-0.01-1.26)$ & $0.15(-0.47-0.76)$ & $0.47(-0.11-1.05)$ & $0.08(-0.55-0.70)$ & $0.30(-0.61-1.21)$ \\
\hline
\end{tabular}

Data are effect size $(95 \% \mathrm{CI})$. The measurement units of all laboratory values for the fasting serum levels of lipids and glucose use mmol/L. All indicators are shown by their respective normal score. BMI: body mass index; TG: triglyceride; LDL: low-density lipoprotein cholesterol; TC: total cholesterol; HDL: high--density lipoprotein cholesterol; FG: fasting serum glucose. 

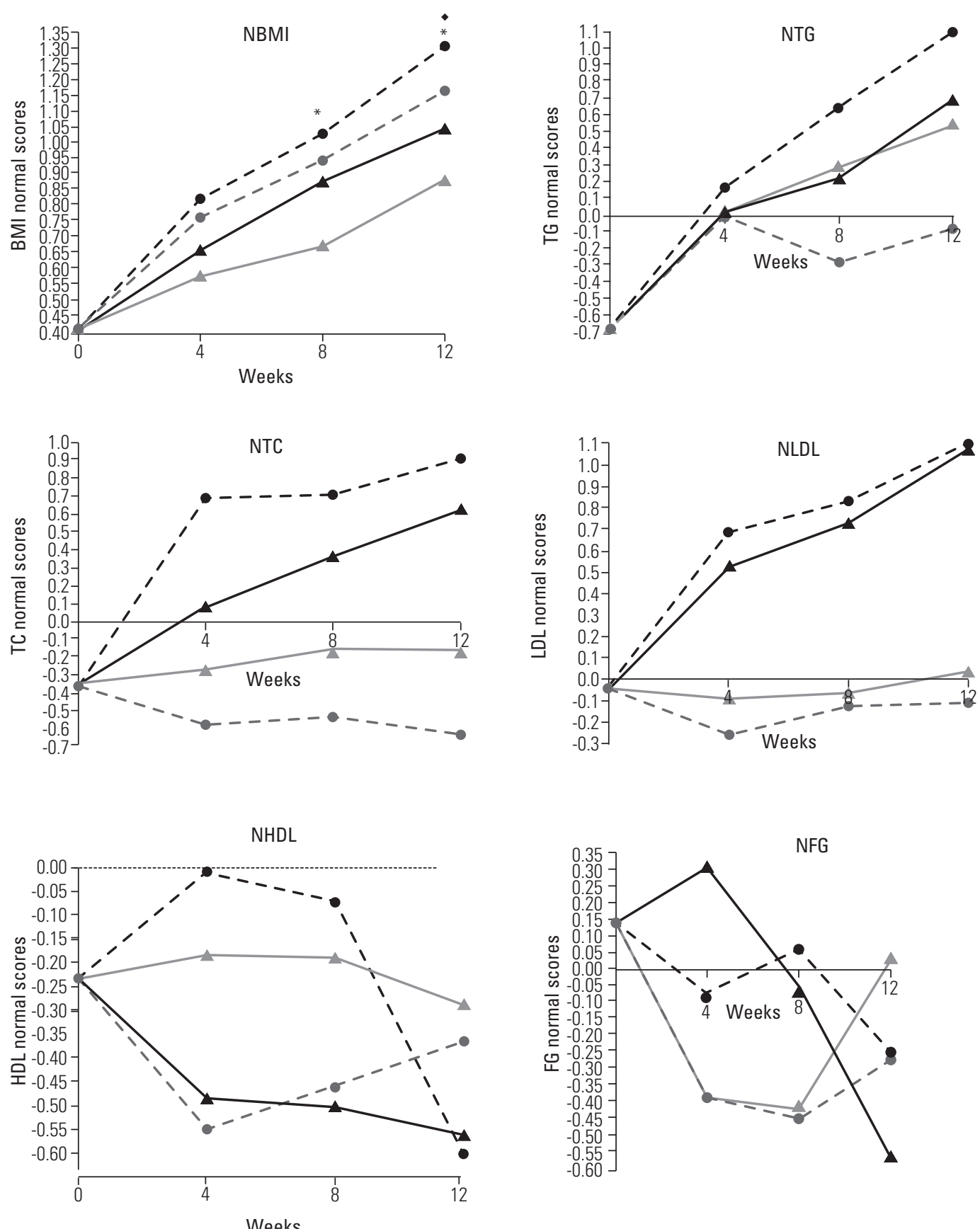

$\rightarrow$ Male Aripiprazole $\quad$-e- Female Aripiprazole

Male Olanzapine

Female Olanzapine

Figure 2. The Evolution of the Metabolic Parameters from Baseline to the 12th Weekend Between-Drug and Between-Sex in Patients with First-Episode Schizophrenia. All indicators are shown by their respective normal score. BMI: body mass index; TG: triglyceride; LDL: Iow-density lipoprotein cholesterol; TC: total cholesterol; HDL: high-density lipoprotein cholesterol; FG: fasting serum glucose. The measurement units of all laboratory values for the fasting serum levels of lipids and glucose use mmol/L. What we are concerned about are the slopes of several subgroups, that is, the increasing or decreasing magnitude. To make the graphics more intuitive, we thereby have moved the curves of the rest of the subgroups parallel, all starting with the baseline values of the MA group. ${ }^{*}$ Sex difference in the aripiprazole group, $p<0.05$. Sex difference in the olanzapine group, $p<0.05$.

The magnitude increasing in MO's NTC lagged behind that of FO during postbaseline visits, resultantly, negative between-sex differences in NTC persisted in patients with olanzapine (Table 3, Figure 2), signifying olanzapine may have an adverse effect of TC on female over male patients with schizophrenia.

\section{High density lipoprotein cholesterol (HDL)}

A negative between-sex difference in baseline NHDL and subsequent positive between-sex differences in postbaseline NHDL elucidated the amplitude decreasing in postbaseline NHDL of females over that of males in patients with aripiprazole (Table 2, Figure 2), 
Table 3. The between-sex and within-sex evolution of the individual metabolic parameter over time in patients with first-episode schizophrenia assigned to 12 weeks treatment with olanzapine

\begin{tabular}{|l|c|c|c|c|c|c|}
\hline & NBMI & NTG & NLDL & NTC & NHDL & NFG \\
\hline Baseline, female & $-0.84(-1.30-0.37)$ & $-0.91(-1.47--0.35)$ & $-0.60(-1.20--0)$ & $-0.50(-1.10-0.10)$ & $0.46(-0.14-1.06)$ & $0.43(-0.13-0.99)$ \\
\hline (The 4th weekend - Baseline), female & $0.41(0.24-0.59)$ & $0.83(0.30-1.36)$ & $0.73(0.28-1.18)$ & $1.05(0.58-1.51)$ & $0.22(-0.30-0.74)$ & $-0.21(-0.89-0.46)$ \\
\hline (The 8th weekend - Baseline), female & $0.62(0.44-0.79)$ & $1.31(0.78-1.84)$ & $0.87(0.42-1.33)$ & $1.07(0.61-1.54)$ & $0.16(-0.36-0.68)$ & $-0.08(-0.76-0.60)$ \\
\hline (The 12th weekend - Baseline), female & $0.89(0.72-1.07)$ & $1.76(1.23-2.29)$ & $1.14(0.68-1.59)$ & $1.27(0.81-1.74)$ & $-0.37(-0.89-0.15)$ & $-0.39(-1.06-0.29)$ \\
\hline Baseline $\times$ (male - female) & $0.85(0.21-1.49)$ & $0.46(-0.32-1.23)$ & $0.28(-0.55-1.10)$ & $0.09(-0.74-0.91)$ & $-0.79(-1.62-0.04)$ & $-0.16(-0.93-0.62)$ \\
\hline The 4th weekend $\times$ (male - female) & $-0.16(-0.40-0.08)$ & $-0.14(-0.87-0.60)$ & $-0.16(-0.79-0.46)$ & $-0.60(-1.24-0.04)$ & $-0.48(-1.19-0.24)$ & $0.37(-0.56-1.31)$ \\
\hline The 8th weekend $\times$ (male - female) & $-0.15(-0.39-0.09)$ & $-0.42(-1.16-0.31)$ & $-0.10(-0.73-0.52)$ & $-0.35(-0.99-0.29)$ & $-0.43(-1.15-0.29)$ & $-0.11(-1.05-0.82)$ \\
\hline The 12th weekend $\times$ (male - female) & $-0.26(-0.50-0.02)$ & $-0.40(-1.14-0.33)$ & $-0.02(-0.65-0.60)$ & $-0.29(-0.93-0.35)$ & $0.04(-0.68-0.76)$ & $-0.31(-1.25-0.62)$ \\
\hline
\end{tabular}

Data are effect size $(95 \% \mathrm{Cl})$. The measurement units of all laboratory values for the fasting serum levels of lipids and glucose use mmol/L. All indicators are shown by their respective normal score. BMI: body mass index; TG: triglyceride; LDL: low-density lipoprotein cholesterol; TC: total cholesterol; HDL: high-density lipoprotein cholesterol; FG: fasting serum glucose.

meaning aripiprazole may have a slightly more adverse effect of HDL metabolism on female patients than that of male patients in our data.

Despite the increases in postbaseline FO's HDL over that of postbaseline MO's HDL at the fourth and the eighth weekend, with the prolongation of treatment time, the former showed a lag behind the latter at the twelfth weekend in patients with olanzapine (Table 3, Figure 2), elucidating olanzapine may have a more adverse effect of HDL metabolism on female patients than male patients.

\section{Fasting glucose (FG)}

The overall downward trend in postbaseline FA's NFG than baseline caused a gradually increasing between-sex difference in postbaseline NFG in patients with aripiprazole (Table 2, Figure 2), meaning there may be an aripiprazole-induced favourable effect of glucose metabolism on female over male patients.

The amplitude increasing in postbaseline FO's NFG than baseline exceeded that of MO group in patients with olanzapine, except for an isolated change that the former lagged behind the latter at the fourth weekend (Table 3, Figure 2), reflecting there may be an olanzapine-induced adverse effect of glucose metabolism on female over male patients.

\section{Discussion}

We have only included those patients being never exposed to any antipsychotic medication prior to the present trial, which facilitated us to directly assess independent effects of antipsychotics on glucose and lipid metabolism and present the precise effect size of incremental metabolic advance between the sexes and between two drugs for each metabolic parameter.

\section{Sex difference in weight gain}

In a study, the aripiprazole-induced change in weight is not associated with baseline weight in schizophrenia patients ${ }^{15}$. In another research, however, aripiprazole is associated with weight gain in patients with baseline underweight, with minimal weight change in patients with baseline normal weight, and with weight loss in patients with baseline overweight ${ }^{16}$. The research by Kasper et al. ${ }^{16}$. supported our finding that aripiprazole may be responsible for weight gain in female schizophrenia patients with low baseline weight. Moreover, the previous reports involving in aripiprazole ${ }^{16-18}$ and olanzapine ${ }^{17,19}$ in line with our findings that both olanzapine and aripiprazole may have an adverse effect of weight on female over male patients strengthen the validity of our interpretations. It is argued that either patients with low baseline BMI or female patients are more biologically vulnerable to metabolic adversities of antipsychotics (such as weight gain) ${ }^{17}$.

\section{Sex difference in lipid metabolism}

Either significant or numerical increase of MA over FA in postbaseline NTG, NTC and NLDL as well as slight decrease of FA over MA in postbaseline NHDL was observed in patients with aripiprazole from our data (Table 2, Figure 2). In general, aripiprazole may have a more favourable effect of lipid metabolism on female patients than male patients. The previous researches ${ }^{2-7}$ supported our findings that both olanzapine and aripiprazole may have an adverse effect of HDL metabolism on female over male schizophrenia patients. In a recent review identifying lipid level in male and female antipsychotic-naïve first-episode psychosis patients, however, the amplitude increasing in HDL and TG of females exceeds the amplitude decreasing in HDL and TG of males after 3-month aripriprazole treatment ${ }^{20}$. Their data from subjects with heterogeneous mental illness and from horizontal rather than vertical comparisons may be one of the reasons why their finding is less consistent with our observations about an aripriprazole-induced adverse effect of HDL and favourable effect of TG on females over males. In an early study identifying lipid level in male and female antipsychotic-naïve first-episode schizophrenia patients from China, triglyceride levels of males were higher than that of females at 8 -week olanzapine treatment ${ }^{21}$. The different angle collecting data, the distinct method analyzing data and the diverse focus reporting data by Wu et al..$^{21}$ may be the reason of this discrepant between-sex result about TG metabolism in schizophrenia patients with olanzapine. Moreover, $\mathrm{Wu}$ et al. did not pay more attention to olanzapine-induced changes in FG and TC in their data, in fact, which can give our observations a side support.

\section{Sex difference in fasting glucose (FG)}

Crawford and DeLisi's data ${ }^{20}$ on FG, TC and LDL support our inferences of between-sex difference in effects of aripiprazole on these indices, although their data on HDL and TG differed from our observations. It is found from our data and others' researches ${ }^{16,17}$ that aripiprazole has the greatest effect on weight gain in those female patients with low baseline BMI. Baseline weight of females was significantly lower than that of males in our data (Table 2). The significant effect of aripiprazole on females' weight in our subjects thus reflected in biochemical indicators in vivo, such as HDL and TG, which may be another reason why the between-sex results of our two indicators differ from theirs ${ }^{20}$. HDL and TG may be more sensitive to the changes in body weight than other lipid markers in schizophrenia patients with antipsychotic treatment.

To our knowledge, we are the first to reveal the sex-dependent differences about both an aripiprazole-induced favourable and an olanzapine-induced adverse effect of glucose and lipid metabolism on female over male antipsychotic-naïve patients with first-episode schizophrenia. 


\section{Connection between glucose and lipid metabolism and weight gain}

The FA group monthly showed the steep rise in postbaseline NBMI, either mild decrease in postbaseline NLDL, NTC, NHDL, NFG or slight increase in postbaseline NTG than baseline in patients with aripiprazole (Table 2, Figure 2). Also, it was not difficult to observe that the adverse effect of aripiprazole on NBMI outnumbered on NTG in all male patients (Table 4).Therefore, the direct effect of aripiprazole on glucose and lipid metabolism may be independent of a change in body weight, especially for female patients with schizophrenia. Our finding is consistent with previously published studies that weight gain was not associated with clinically significant changes in glucose and lipid in the patients with aripiprazole ${ }^{18,22}$. The observation that aripiprazole causes weight gain but does not affect the level of circulating glucose and lipid has substantial clinical and research implications, which mechanism will be explored in deep in our next paper.

Numerous clinical studies ${ }^{23}$ confirmed the association among olanzapine administration, weight gain and the aggravation of glucose and lipid profiles in the patients with schizophrenia. A study in individuals with schizophrenia spectrum disorders on long term clozapine or olanzapine treatment within the community reported females developed worse metabolic profiles than males ${ }^{24}$. The findings from our data not only support these observations, but also further reveal sex difference in metabolic adverse effect of olanzapine on patients with first-onset schizophrenia. Given that female patients have significantly higher plasma concentrations of olanzapine ${ }^{25,26}$, and require less dose of olanzapine treatment than male patients ${ }^{27}$, our data also confirm that the FO group shows the highest level of glucose and lipid deterioration in the four subgroups (Figure 2), we thereby advocate the preventive use of the adjunctive agent with antiobesity and antidiabetic mechanism at initial exposure to olanzapine against metabolic adverse effects secondary to olanzapine administration, especially for female patients with schizophrenia. Another good alternative may be lower doses, longer dosing intervals of olanzapine for female than for male patients with schizophrenia. Certainly, it still requires further support from large sample, multi-ethnic, randomized controlled trials and the longer-term assessment showing the trajectory of metabolic changes to consider this treatment strategy as a routine adjuvant therapy to olanzapine administration in future revision of therapeutic guidelines.

\section{Conclusion}

Despite the unknown exact pharmacological mechanisms inducing different pathophysiological processes underlying several mutually independent adverse effects (such as weight gain, glucose metabolism abnormalities and dyslipidemia), establishing the relative contributions of antipsychotics to sex can provide psychiatrists with the more targeted SAGs recommends so that they can better tailor treatment to individual sex-differential physiological characteristics. We hope that some individualized treatment strategies to prescribe antipsychotic drugs will emerge in the future clinical psychiatry practice, especially one distinguishing between sexes. Furthermore, further pathophysiology characterization underlying some sexdifferential effects of aripiprazole and olanzapine on metabolic profiles also will provide new clues to develop new pharmacological options minimizing SGAs-induced metabolic adverse effects. A better understanding of mechanistic connections among glucose metabolism abnormalities, lipid disturbances and weight gain induced by SGAs may ultimately allow identification of risk factors and prevention of those metabolic adverse responses with adjunctive approaches in schizophrenia patients.

\section{Limits}

First, the sample size of the current study may not be optimal but should be sufficient to our conclusions that may guide clinical practice. Second, it is seen from Stable 2 (available upon request to the authors) that there are two underpowered indicators in the aripiprazole group and the olanzapine group, respectively. However, any underpowered indicator does not reduce the reliability of our inference, because sex differences of glucose and lipid identified by us are based not only on their statistical significance but also on their patterns in numerical change. In fact, the increase in the probability of a type II error resulted from underpowered data precisely strengthens our inferences of sex difference in glucose and lipid metabolism.

\section{Disclosure of interests}

The authors declared no conflicts of interest with respect to the research, authorship and/or publication of this article.

\section{Role of the funding source}

This research was mostly supported by the Science and Technology Projects of the Health and Family Planning Commission of Jiangxi Province under Grant (number 20157041 to Dr. ZHou); and partially by the National Natural Science Foundation of China under Grant (number 81360211 to $\mathrm{Dr}$. Hu). The funder of the study had no role in study design, data collection, data analysis, data interpretation, writing of the report, or the decision to submit the paper for publication.

\section{Trial Registration}

Chinese Clinical Trial Registry, ChiCTR-OPC-16009742, http://www.who.int/ictrp/network/primary/en/index.html.

Table 4. The between-drug and within-drug evolution of the individual metabolic parameter over time in male patients with first-episode schizophrenia assigned to 12 weeks treatment with aripiprazole or olanzapine

\begin{tabular}{|c|c|c|}
\hline & NBMI & NTG \\
\hline Baseline, aripiprazole & $0.41(-0.05-0.88)$ & $-0.66(-1.24--0.09)$ \\
\hline (The 4th weekend - Baseline), aripiprazole & $0.17(0.01-0.32)$ & $0.70(0.22-1.18)$ \\
\hline (The 8th weekend - Baseline), aripiprazole & $0.26(0.11-0.42)$ & $0.95(0.47-1.43)$ \\
\hline (The 12th weekend - Baseline), aripiprazole & $0.47(0.32-0.63)$ & $1.21(0.73-1.69)$ \\
\hline Baseline $\times$ (olanzapine - aripiprazole) & $-0.40(-1.06-0.26)$ & $0.21(-0.60-1.03)$ \\
\hline The 4th weekend $\times$ (olanzapine - aripiprazole) & $0.08(-0.13-0.30)$ & $0(-0.68-0.68)$ \\
\hline The 8th weekend $\times$ (olanzapine - aripiprazole) & $0.21(-0.01--0.42)$ & $-0.07(-0.74-0.61)$ \\
\hline The 12th weekend $\times$ (olanzapine - aripiprazole) & $0.16(-0.06--0.38)$ & $0.14(-0.54-0.82)$ \\
\hline
\end{tabular}

Data are effect size $(95 \% \mathrm{CI})$. The measurement units of all laboratory values for the fasting serum levels of lipids and glucose use mmol/L. All indicators are shown by their respective normal score. BMI: body mass index; TG: triglyceride. 


\section{References}

1. Clayton JA, Collins FS. Policy: NIH to balance sex in cell and animal studies. Nature. 2014;509(7500):282-3.

2. Bobes J, Arango C, Aranda P, Carmena R, Garcia-Garcia M, Rejas J, et al. Cardiovascular and metabolic risk in outpatients with schizophrenia treated with antipsychotics: results of the CLAMORS Study. Schizophr Res. 2007;90(1-3):162-73.

3. Bobes J, Arango C, Aranda P, Carmena R, Garcia-Garcia M, Rejas J, et al. Cardiovascular and metabolic risk in outpatients with schizoaffective disorder treated with antipsychotics: results from the CLAMORS study. Eur Psychiatry. 2012;27(4):267-74.

4. Hägg S, Lindblom Y, Mjörndal T, Adolfsson R. High prevalence of the metabolic syndrome among a Swedish cohort of patients with schizophrenia. Int Clin Psychopharmacol. 2006;21(2):93-8.

5. Huang MC, Lu ML, Tsai CJ, Chen PY, Chiu CC, Jian DL, et al. Prevalence of metabolic syndrome among patients with schizophrenia or schizoaffective disorder in Taiwan. Acta Psychiatr Scand. 2009;120(4):274-80.

6. McEvoy JP, Meyer JM, Goff DC, Nasrallah HA, Davis SM, Sullivan L, et al. Prevalence of the metabolic syndrome in patients with schizophrenia: baseline results from the Clinical Antipsychotic Trials of Intervention Effectiveness (CATIE) schizophrenia trial and comparison with national estimates from NHANES III. Schizophr Res. 2005;80(1):19-32.

7. Sugawara N, Yasui-Furukori N, Sato Y, Umeda T, Kishida I, Yamashita $\mathrm{H}$, et al. Prevalence of metabolic syndrome among patients with schizophrenia in Japan. Schizophr Res. 2010;123(2-3):244-50.

8. Lee JS, Kwon JS, Kim D, Kim SW, Kim JJ, Kim JH, et al. Prevalence of Metabolic Syndrome in Patients with Schizophrenia in Korea: A Multicenter Nationwide Cross-Sectional Study. Psychiatry Investig. 2017;14(1):44-50

9. Rezaei O, Khodaie-Ardakani MR, Mandegar MH, Dogmehchi E, Goodarzynejad H. Prevalence of metabolic syndrome among an Iranian cohort of inpatients with schizophrenia. Int J Psychiatry Med. 2009;39(4):451-62.

10. Li Q, Chen D, Liu T, Walss-Bass C, de Quevedo JL, Soares JC, et al. Sex Differences in Body Mass Index and Obesity in Chinese Patients with Chronic Schizophrenia. J Clin Psychopharmacol. 2016;36(6):643-8.

11. Chen S, Broqueres-You D, Yang G, Wang Z, Li Y, Yang F, et al. Male sex may be associated with higher metabolic risk in first-episode schizophrenia patients: A preliminary study. Asian J Psychiat. 2016;21:25-30.

12. General Assembly of the World Medical Association. World Medical Association Declaration of Helsinki: ethical principles for medical research involving human subjects. J Am Coll Dent. 2014;81(3):14-8.

13. American Psychiatric Association. Diagnostic and statistical manual of mental disorders. 5th ed. Washington, DC: American Psychiatric Association; 2013.
14. Kay SR, Fiszbein A, Opler LA. The Positive and Negative Syndrome Scale (PANSS) for schizophrenia. Schizophr Bull. 1987;13(2):261-76.

15. Pigott TA, Carson WH, Saha AR, Torbeyns AF, Stock EG, Ingenito GG. Aripiprazole for the prevention of relapse in stabilized patients with chronic schizophrenia: a placebo-controlled 26-week study. J Clin Psychiatr. 2003;64(9):1048-56.

16. Kasper S, Lerman MN, McQuade RD, Saha A, Carson WH, Ali M, et al. Efficacy and safety of aripiprazole vs. haloperidol for long-term maintenance treatment following acute relapse of schizophrenia. Int J Neuropsychopharmacol. 2003;6(4):325-37.

17. Lee SY, Park MH, Patkar AA, Pae CU. A retrospective comparison of BMI changes and the potential risk factors among schizophrenic inpatients treated with aripiprazole, olanzapine, quetiapine or risperidone. Prog Neuropsychopharmacol Biol Psychiatry. 2011;35(2):490-6.

18. McIntyre RS, McElroy SL, Eudicone JM, Forbes RA, Carlson BX, Baker RA. A 52-week, double-blind evaluation of the metabolic effects of aripiprazole and lithium in bipolar I disorder. Prim Care Companion CNS Disord. 2011;13(6):PCC.11m01182.

19. Jain S, Bhargava M, Gautam S. Weight gain with olanzapine: Drug, gender or age? Indian J Psychiatry. 2006;48(1):39-42.

20. Crawford MB, DeLisi LE. Issues related to sex differences in antipsychotic treatment. Curr Opin Psychiatry. 2016;29(3):211-7.

21. Wu RR, Zhao JP, Zhai JG, Guo XF, Guo WB. Sex difference in effects of typical and atypical antipsychotics on glucose-insulin homeostasis and lipid metabolism in first-episode schizophrenia. J Clin Psychopharmacol. 2007;27(4):374-9.

22. Kemp DE, De Hert M, Rahman Z, Fyans P, Eudicone JM, Marler SV, et al. Investigation into the long-term metabolic effects of aripiprazole adjunctive to lithium, valproate, or lamotrigine. J Affect Disord. 2013;148(1):84-91.

23. Khanna P, Suo T, Komossa K, Ma H, Rummel-Kluge C, El-Sayeh HG, et al. Aripiprazole versus other atypical antipsychotics for schizophrenia. Cochrane Database Syst Rev. 2014;(2):CD006569.

24. Kraal AZ, Ward KM, Ellingrod VL. Sex Differences in Antipsychotic Related Metabolic Functioning in Schizophrenia Spectrum Disorders. Psychopharmacol Bull. 2017;47(2):8-21.

25. Gex-Fabry M, Balant-Gorgia AE, Balant LP. Therapeutic drug monitoring of olanzapine: the combined effect of age, gender, smoking, and comedication. Ther Drug Monit. 2003;25(1):46-53.

26. Weiss U, Marksteiner J, Kemmler G, Saria A, Aichhorn W. Effects of age and sex on olanzapine plasma concentrations. J Clin Psychopharmacol. 2005;25(6):570-4.

27. Eugene AR, Masiak J. A pharmacodynamic modelling and simulation study identifying gender differences of daily olanzapine dose and dopamine D2-receptor occupancy. Nord J Psychiatry. 2017;71(6):417-24. 


\section{Sex-differential effects of olanzapine vs. aripiprazole on glucose and lipid metabolism in first-episode schizophrenia}

XUE-MEI ZHOU 1

MaO-Rong Hu2

http://orcid.org/0000-0002-8541-2893

MeI-Yu Gong1

http://orcid.org/0000-0002-9092-3006

Xue-Liang Zou ${ }^{1}$

http://orcid.org/0000-0003-0688-1550

ZHI-MIN YU1

http://orcid.org/0000-0002-8732-4811

1 Jiangxi Mental Health Centre, Nanchang, Jiangxi, China.

2 The First Affiliated Hospital of Nanchang University, Nanchang, Jiangxi, China.

ZHou XM et al. / Arch Clin Psychiatry. 2019;46(2):33-9

Received: 06/29/2018 - Accepted: 04/02/2019

DOl: 10.1590/0101-60830000000189

On Table 4, where is written:

Table 4. The between-drug and within-drug evolution of the individual metabolic parameter over time in male patients with first-episode schizophrenia assigned to 12 weeks treatment with aripiprazole or olanzapine

\begin{tabular}{|c|c|c|}
\hline & NBMI & NTG \\
\hline Baseline, female & $0.41(-0.05-0.88)$ & $-0.66(-1.24--0.09)$ \\
\hline (The 4th weekend - Baseline), female & $0.17(0.01-0.32)$ & $0.70(0.22-1.18)$ \\
\hline (The 8th weekend - Baseline), female & $0.26(0.11-0.42)$ & $0.95(0.47-1.43)$ \\
\hline (The 12th weekend - Baseline), female & $0.47(0.32-0.63)$ & $1.21(0.73-1.69)$ \\
\hline Baseline $\times($ male - female $)$ & $-0.40(-1.06-0.26)$ & $0.21(-0.60-1.03)$ \\
\hline The 4 th weekend $\times$ (male - female) & $0.08(-0.13-0.30)$ & $0(-0.68-0.68)$ \\
\hline The 8th weekend $\times$ (male - female) & $0.21(-0.01--0.42)$ & $-0.07(-0.74-0.61)$ \\
\hline The 12 th weekend $\times$ (male - female) & $0.16(-0.06--0.38)$ & $0.14(-0.54-0.82)$ \\
\hline
\end{tabular}

Data are effect size $(95 \% \mathrm{CI})$. The measurement units of all laboratory values for the fasting serum levels of lipids and glucose use $\mathrm{mmol} / \mathrm{L}$. All indicators are shown by their respective normal score. BMI: body mass index; TG: triglyceride.

It should be read:

Table 4. The between-drug and within-drug evolution of the individual metabolic parameter over time in male patients with first-episode schizophrenia assigned to 12 weeks treatment with aripiprazole or olanzapine

\begin{tabular}{|c|c|c|}
\hline & NBMI & NTG \\
\hline Baseline, aripiprazole & $0.41(-0.05-0.88)$ & $-0.66(-1.24--0.09)$ \\
\hline (The 4th weekend - Baseline), aripiprazole & $0.17(0.01-0.32)$ & $0.70(0.22-1.18)$ \\
\hline (The 8th weekend - Baseline), aripiprazole & $0.26(0.11-0.42)$ & $0.95(0.47-1.43)$ \\
\hline (The 12th weekend - Baseline), aripiprazole & $0.47(0.32-0.63)$ & $1.21(0.73-1.69)$ \\
\hline Baseline $\times$ (olanzapine - aripiprazole) & $-0.40(-1.06-0.26)$ & $0.21(-0.60-1.03)$ \\
\hline The 4th weekend $\times$ (olanzapine - aripiprazole) & $0.08(-0.13-0.30)$ & $0(-0.68-0.68)$ \\
\hline The 8th weekend $\times$ (olanzapine - aripiprazole) & $0.21(-0.01--0.42)$ & $-0.07(-0.74-0.61)$ \\
\hline The 12 th weekend $\times$ (olanzapine - aripiprazole) & $0.16(-0.06--0.38)$ & $0.14(-0.54-0.82)$ \\
\hline
\end{tabular}

Data are effect size $(95 \% \mathrm{CI})$. The measurement units of all laboratory values for the fasting serum levels of lipids and glucose use mmol/L. All indicators are shown by their respective normal score. BMI: body mass index; TG: triglyceride. 